\title{
Orbital Stability of Solitary Waves for Generalized Klein-Gordon-Schrödinger Equations
}

\author{
Wenhui Qi, Guoguang Lin \\ School of Mathematics and Statistic, Yunnan University, Kunming, China \\ E-mail:gglin@ynu.edu.cn \\ Received May 30, 2011; revised June 13, 2011; accepted June 20, 2011
}

\begin{abstract}
This paper concerns the orbital stability for exact solitary waves of the Generalized Klein-Gordon-Schrödinger equations. Since the abstract results of Grillakis et al. [1,2] can not be applied directly, we can extend the abstract stability theory and use the detailed spectral analysis to obtain the stability of the solitary waves.
\end{abstract}

Keywords: Solitary Waves, Stability, Klein-Gordon-Schrödinger Equations

\section{Introduction}

In this paper, we consider the the stability for the exact solitary waves of the Generalized Klein-Gordon-Schrödinger equations

$$
\left\{\begin{array}{c}
i \psi_{t}+\alpha \psi_{x x}=-|\varphi|^{p}|\psi|^{p-2} \psi \\
\varphi_{t t}-\varphi_{x x}+M^{2} \varphi=|\psi|^{p}|\varphi|^{p-2} \varphi
\end{array} \quad x \in R\right.
$$

which describe a classical model of interaction of nucleon field with a meson field [3]. Here $\psi$ is a complex scalar nucleon field, $\varphi$ is a real meson field, $\mathrm{M}$ is the mass of a meson. By applying the abstract stability theory and detailed spectral analysis in [4-6], we obtain the orbital stability of the solitary waves.

This paper is organized as follows: in Section 2, we state the results of the existence of the exact solitary waves; in Section 3, we state the assumptions and the stability results.

\section{The Exact Solitary Waves}

Consider the following system

$$
\left\{\begin{array}{c}
i \psi_{t}+\alpha \psi_{x x}=-|\varphi|^{p}|\psi|^{p-2} \psi \\
\varphi_{t t}-\varphi_{x x}+M^{2} \varphi=|\psi|^{p}|\varphi|^{p-2} \varphi
\end{array} \quad x \in R\right.
$$

Let

$$
\left\{\begin{array}{l}
\psi(x, t)=\mathrm{e}^{i \omega t} \mathrm{e}^{i c(x-c t)} u(x-c t) \\
\varphi(x, t)=v(x-c t)
\end{array}\right.
$$

be the solitary waves of (2.1).

Put (2.2) into (2.1) and suppose $u, u^{\prime \prime}, v, v^{\prime \prime} \rightarrow 0$,as $x \rightarrow \infty$,we obtain

$$
\left\{\begin{array}{l}
(2 \alpha-1) c u^{\prime}=0 \\
\alpha u^{\prime \prime}+\left(c^{2}-\omega-\alpha c^{2}\right) u+|v|^{p}|u|^{p-2} u=0 \\
\left(c^{2}-1\right) v^{\prime \prime}+M^{2} v-|u|^{p}|v|^{p-2} v=0
\end{array}\right.
$$

Let

$$
\alpha=\frac{1}{2}, u=k v
$$

satisfy (2.3) with constant $k \neq 0$ determined later, then we have

$$
\left\{\begin{array}{l}
u^{\prime \prime}+\left(c^{2}-2 \omega\right) u+\frac{2}{|k|^{p}}|u|^{2 p-2} u=0 \\
\left(c^{2}-1\right) v^{\prime \prime}+M^{2} v-|k|^{p}|v|^{2 p-2} v=0
\end{array}\right.
$$

Let $u=c_{1} \operatorname{sech}^{\frac{1}{p-1}} c_{2} x$ satisfy (2.4)-(2.5) and constants $c_{1}, c_{2}$ will be determined later, then we obtain

$$
\begin{aligned}
& k^{2}=2\left(1-c^{2}\right), c_{2}^{2}=\frac{M^{2}(1-p)^{2}}{1-c^{2}}=\left(2 \omega-c^{2}\right)(1-p)^{2} \\
& c_{1}^{2 p-2}=2^{\frac{p-2}{2}} p\left(2 \omega-c^{2}\right)\left(1-c^{2}\right)^{\frac{p}{2}}
\end{aligned}
$$

Thus 


$$
\begin{gathered}
u(x)=\left[2^{\frac{p-2}{2}} p\left(2 \omega-c^{2}\right)\left(1-c^{2}\right)^{\frac{p}{2}}\right]^{\frac{1}{2 p-2}} \\
\cdot \operatorname{sech}^{\frac{1}{p-1}}\left(\sqrt{2 \omega-c^{2}}(p-1) x\right) \\
v(x)=\frac{\left[2^{\frac{p-2}{2}} p\left(2 \omega-c^{2}\right)\left(1-c^{2}\right)^{\frac{p}{2}}\right]^{\frac{1}{2 p-2}}}{\sqrt{2\left(1-c^{2}\right)}} \\
\cdot \operatorname{sech}^{\frac{1}{p-1}}\left(\sqrt{2 \omega-c^{2}}(p-1) x\right)
\end{gathered}
$$

Finally, we have

Theorem 1. For any real constants $\omega, c, p, M$ satisfying

$$
0<c<1, \omega>\frac{1}{2}, \quad p>1, M>0
$$

there exist solitary wave of (2.1) in the form of (2.2),with $u, v$ satisfying (2.6).

\section{Main Results}

Rewrite Equation (2.1) as

$$
\left\{\begin{array}{l}
i \psi_{t}+\frac{1}{2} \psi_{x x}+|\varphi|^{p}|\psi|^{p-2} \psi=0 \\
\varphi_{t}=n \quad, \quad x \in R \\
n_{t}=\varphi_{x x}-M^{2} \varphi+|\psi|^{p}|\varphi|^{p-2} \varphi
\end{array}\right.
$$

Let $\boldsymbol{u}=\left(\begin{array}{l}\phi \\ \psi \\ n\end{array}\right)$, and the function space in which we

shall work is $X=H_{\text {real }}^{1}(R) \times H_{\text {complex }}^{1}(R) \times L_{\text {real }}^{2}$, with inner product

$$
(\boldsymbol{f}, \boldsymbol{g})=\operatorname{Re} \int_{R}\left(f_{1} g_{1}+f_{1 x} g_{1 x}+f_{2} \bar{g}_{2}+f_{2 x} \bar{g}_{2 x}+f_{3} g_{3}\right) \mathrm{d} x,
$$

$f, g \in X$

The dual space of $X$ is $X^{*}=H_{\text {real }}^{-1} \times H_{\text {complex }}^{-1} \times L_{\text {real }}^{2}$, there is a natural isomorphism $I: X \rightarrow X^{*}$ defined by

$$
\langle\text { If }, g\rangle=(\boldsymbol{f}, \boldsymbol{g})
$$

where $<\cdot, \cdot>$ denotes the pairing between $X$ and $X^{*}$.

$$
\langle\boldsymbol{f}, \boldsymbol{g}\rangle=\operatorname{Re} \int_{R}\left(f_{1} g_{1}+f_{2} \bar{g}_{2}+f_{3} g_{3}\right) \mathrm{d} x
$$

By (3.2)-(3.4), it is obvious

$$
I=\left(\begin{array}{ll}
1 & \\
& 1-\frac{\partial^{2}}{\partial x^{2}}
\end{array}\right)
$$

Because the stability in view here refers to perturbations of the solitary-wave profile itself, a study of the initial-value problem for (1.1) is necessary.

Lemma 1. Let $\boldsymbol{u}_{0} \in H_{\text {real }}^{1}(R) \times H_{\text {complex }}^{1}(R) \times L_{\text {real }}^{2}$, there exists $T_{*}=T_{*}\left(\left\|\vec{u}_{0}\right\|\right)>0$ and a unique solution $\boldsymbol{u} \in C\left(\left[0, T_{*}\right) ; H^{1} \times H^{1} \times L^{2}\right), \boldsymbol{u}(0)=\boldsymbol{u}_{0}$. In addition, either $T_{*}=\infty$ or $\|\boldsymbol{u}(x, t)\|_{X} \rightarrow \infty\left(t \rightarrow T_{*}\right)$.

Let $T_{1}, T_{2}$ be one-parameter groups of unitary operator on $X$ defined by

$$
\begin{gathered}
T_{1}\left(s_{1}\right) \boldsymbol{u}(\cdot)=\boldsymbol{u}\left(\cdot-s_{1}\right), \boldsymbol{u}(\cdot) \in X, s \in R \\
T_{2}\left(s_{2}\right) \boldsymbol{u}(\cdot)=\left(\varphi(\cdot), \mathrm{e}^{i s_{2}} \psi(\cdot), n(\cdot)\right), \boldsymbol{u}(\cdot) \in X, s \in R
\end{gathered}
$$

Obviously

$$
T_{1}^{\prime}(0)=\left(\begin{array}{rrr}
-\frac{\partial}{\partial x} & & \\
& -\frac{\partial}{\partial x} & \\
& & -\frac{\partial}{\partial x}
\end{array}\right), \quad T_{2}^{\prime}(0)=\left(\begin{array}{lll}
0 & & \\
& i & \\
& & 0
\end{array}\right)
$$

It follows from Theorem 1 and (3.1) that there exist solitary waves $T_{1}(c t) T_{2}(\omega t)\left(\varphi_{\omega, c}(x), \psi_{\omega, c}(x), n_{\omega, c}(x)\right)$ with $\varphi_{\omega, c}(x), \psi_{\omega, C}(x), n_{\omega, c}(x)$ defined by

$$
\left\{\begin{array}{l}
\varphi_{\omega, c}(x)=v(x) \\
\psi_{\omega, c}(x)=e^{i c x} u(x) \\
n_{\omega, c}(x)=-c v^{\prime}(x)
\end{array}\right.
$$

Let

$$
\Phi_{\omega, c}(x)=\left(\varphi_{\omega, c}(x), \psi_{\omega, c}(x), n_{\omega, c}(x)\right)
$$

In this and the following sections, we shall consider the orbital stability of solitary waves

$T_{1}(c t) T_{2}(\omega t) \Phi_{\omega, c}(x)$ of (3.1). Note that Equation (3.1) is invariant under $T_{1}(\cdot)$ and $T_{2}(\cdot)$,we define the orbital stability as follows:

Definition 1. The solitary wave $T_{1}(c t) T_{2}(\omega t) \Phi_{\omega, c}(x)$ is orbitally stable if for all $\varepsilon>0$ there exists $\delta>0$ with the following property. If $\left\|\boldsymbol{u}_{0}-\Phi_{\omega, c}\right\|_{X}<\delta$ and $\boldsymbol{u}(t)$ is a solution of (3.1) in some interval $\left[0, t_{0}\right)$ with $\boldsymbol{u}(0)=\boldsymbol{u}_{0}$, then $\boldsymbol{u}(t)$ can be continued to a solution in 
$0 \leq t<+\infty$, and

$$
\sup _{0<t<+\infty} \inf _{s_{1} \in R} \inf _{s_{2} \in R}\left\|\boldsymbol{u}(t)-T_{1}\left(s_{1}\right) T_{2}\left(s_{2}\right) \Phi_{\omega, c}(x)\right\|_{X}<\varepsilon
$$

Otherwise $T_{1}(c t) T_{2}(\omega t) \Phi_{\omega, c}(x)$ is called orbitally unstable.

So long as $\omega, c$ are fixed we write $\varphi, \psi, n$ for $\varphi_{\omega, c}(x), \psi_{\omega, c}(x), n_{\omega, c}(x)$.

Define

$$
\begin{gathered}
E(\boldsymbol{u})=\int_{R}\left(\frac{1}{2}|n|^{2}+\frac{1}{2}\left|\varphi_{x}\right|^{2}+\frac{1}{2} M^{2}|\varphi|^{2}+\frac{1}{4}\left|\psi_{x}\right|^{2}\right. \\
\left.-\frac{1}{p}|\varphi|^{p}|\psi|^{p}\right) \mathrm{d} x \\
Q_{1}(\boldsymbol{u})=\frac{1}{2} \int_{R}\left(n_{x} \varphi-\varphi_{x} n\right) \mathrm{d} x+\frac{1}{2} \operatorname{Im} \int_{R} \psi_{x} \bar{\psi} \mathrm{d} x \\
Q_{2}(\boldsymbol{u})=-\frac{1}{2} \int_{R}|\psi|^{2} \mathrm{~d} x
\end{gathered}
$$

It is easy to verify that $E(\boldsymbol{u}), Q_{1}(\boldsymbol{u})$ and $Q_{2}(\boldsymbol{u})$ are invariant under $T_{1}, T_{2}$, and formally conserved under the flow of (3.1). Namely

$$
\begin{aligned}
& E\left(T_{1}\left(s_{1}\right) T_{2}\left(s_{2}\right) \boldsymbol{u}\right)=E(\boldsymbol{u}), \text { for } \text { any } s_{1}, s_{2} \in R \\
& Q_{1}\left(T_{1}\left(s_{1}\right) T_{2}\left(s_{2}\right) \boldsymbol{u}\right)=Q_{1}(\boldsymbol{u}), \text { for } \text { any } s_{1}, s_{2} \in R \\
& Q_{2}\left(T_{1}\left(s_{1}\right) T_{2}\left(s_{2}\right) \boldsymbol{u}\right)=Q_{2}(\boldsymbol{u}), \text { for } \text { any } s_{1}, s_{2} \in R
\end{aligned}
$$

and for any $t \in R, \boldsymbol{u}(t)$ is a flow of (3.1)

$$
\begin{aligned}
& E(\boldsymbol{u}(t))=E(\boldsymbol{u}(0)), Q_{1}(\boldsymbol{u}(t))=Q_{1}(\boldsymbol{u}(0)), \\
& Q_{2}(\boldsymbol{u}(t))=Q_{2}(\boldsymbol{u}(0))
\end{aligned}
$$

Note that Equation (3.1) can be written as the following Hamiltonian system

$$
\frac{\mathrm{d} \boldsymbol{u}}{\mathrm{d} t}=J E^{\prime}(\boldsymbol{u})
$$

where $J$ is a skew-symmetric linear operator, $E$ is a functional (the energy).

However, by (2.4)-(2.6), we have

$$
E^{\prime}\left(\phi_{\omega, c}\right)-c Q_{1}^{\prime}\left(\phi_{\omega, c}\right)-\omega Q_{2}^{\prime}\left(\phi_{\omega, c}\right)=0
$$

where $E^{\prime}, Q^{\prime}$ and $Q_{2}^{\prime}$ are the Frechet derivatives of $E$, $Q_{1}$ and $Q_{2}$, with

$$
\begin{aligned}
& E^{\prime}(\boldsymbol{u})=\left(\begin{array}{c}
-\varphi_{x x}+M^{2} \varphi-|\psi|^{p}|\varphi|^{p-2} \varphi \\
-\frac{1}{2} \psi_{x x}-|\varphi|^{p}|\psi|^{p-2} \psi \\
n
\end{array}\right) \\
& Q_{1}^{\prime}(\boldsymbol{u})=\left(\begin{array}{c}
n_{x} \\
-i \psi_{x} \\
-\varphi_{x}
\end{array}\right), \quad Q_{2}^{\prime}(\boldsymbol{u})=\left(\begin{array}{c}
0 \\
-\psi \\
0
\end{array}\right)
\end{aligned}
$$

Define an operator from $X$ to $X^{*}$

$$
H_{\omega, c} \boldsymbol{\varphi}=E^{\prime \prime}\left(\phi_{\omega, c}\right)-c Q_{1}^{\prime \prime}\left(\phi_{\omega, c}\right)-\omega Q_{2}^{\prime \prime}\left(\phi_{\omega, c}\right)
$$

with $\boldsymbol{y}=\left(y_{1}, y_{2}, y_{3}\right) \in X$, and

$$
H_{\omega, c} \psi=\left(\begin{array}{c}
\left(-\frac{\partial}{\partial x^{2}}+M^{2}-(p-1)|\varphi|^{p-2}|\psi|^{p}\right) y_{1}-\frac{p}{2}|\varphi \psi|^{p-2} \varphi(\psi+\bar{\psi}) y_{2}-c y_{3 x} \\
-p|\varphi \psi|^{p-2} \varphi \psi y_{1}+\left[-\frac{1}{2} \frac{\partial}{\partial x^{2}}-\frac{p}{2}|\varphi|^{p}|\psi|^{p-2}-\frac{p-2}{2}|\varphi|^{p}|\psi|^{p-4} \psi^{2}+i c \frac{\partial}{\partial x}+\omega\right] y_{2} \\
c y_{1 x}+y_{3}
\end{array}\right)
$$

Observe that $H_{\omega, c}$ is self-adjoint in the sense that $H_{\omega, c}^{*}=H_{\omega, c}$ This means that $I^{-1} H_{\omega, c}$ is a bounded selfadjoint operator on $X$. The spectrum of $H_{\omega, c}$ consists of the real numbers $\lambda$ such that $H_{\omega, c}-\lambda I$ is not invertible. We claim that $\lambda=0$ belongs to the spectrum of $H_{\omega, c}$. By (3.11-3.15), it is easy to prove that

$$
\begin{aligned}
& H_{\omega, c} T_{1}^{\prime}(0) \Phi_{\omega, c}(x)=0 \\
& H_{\omega, c} T_{2}^{\prime}(0) \Phi_{\omega, c}(x)=0
\end{aligned}
$$

Let

$$
Z=\left\{k_{1} T_{1}^{\prime}(0) \Phi_{\omega, c}(x)+k_{2} T_{2}^{\prime}(0) \Phi_{\omega, c}(x) / k_{1}, k_{2} \in R\right\}
$$

By (3.16), $Z$ is contained in the kernel of $H_{\omega, c}$.

Assumption 1. (Spectral decomposition of $H_{\omega, c}$ )

The space $X$ is decomposed as a direct sum

$$
X=N+Z+P
$$

where $Z$ is defined above, $\mathrm{N}$ is a finite-dimensional subspace such that

$$
\left\langle H_{\omega, c} \boldsymbol{u}, \boldsymbol{u}\right\rangle<0 \quad \text { for } \quad 0 \neq \boldsymbol{u} \in N
$$

and $P$ is a closed subspace such that

$$
\left\langle H_{\omega, c} \boldsymbol{u}, \boldsymbol{u}\right\rangle \geq \delta\|\boldsymbol{u}\|_{X}^{2} \text { for } \boldsymbol{u} \in P
$$


with some constant $\delta>0$ independent of $\boldsymbol{u}$.

We define $d(\omega, c): R \times R \rightarrow R$ by

$$
d(\omega, c)=E\left(\phi_{\omega, c}\right)-c Q_{1}\left(\phi_{\omega, c}\right)-\omega Q_{2}\left(\phi_{\omega, c}\right)
$$

and define $d^{\prime \prime}(\omega, c)$ to be the Hessian of function $d$. It is a symmetric bilinear form. In addition, we use $p\left(d^{\prime \prime}\right)$ to express the numbers of positive eigenvalue of $d^{\prime \prime}$ and $n\left(H_{\omega, c}\right)$ to express the numbers of negative eigenvalue of $H_{\omega, c}$.

Theorem 2. Suppose that there exist three function $E(\boldsymbol{u}), Q_{1}(\boldsymbol{u}), Q_{2}(\boldsymbol{u})$ satisfying (3.11) and (3.12), and solitary waves $T_{1}(c t) T_{2}(\omega t) \Phi_{\omega, c}(x)$ satisfying (3.14). Moreover, suppose that the operator $H_{\omega, c}$ given by (3.15) satisfies Assumption 1. If $d(\omega, c)$ is non-degenerative, $1<p<3$ and $p\left(d^{\prime \prime}\right)=n\left(H_{\omega, c}\right)$, then solitary waves $T_{1}(c t) T_{2}(\omega t) \Phi_{\omega, c}(x)$ are orbitally stable.

Proof. According to (3.8)-(3.15), we only need to prove that Assumption 1 and $p\left(d^{\prime \prime}\right)=n\left(H_{\omega, c}\right)$ hold.

First of all, we prove that Assumption 1 hold and $n\left(H_{\omega, c}\right)=1$

For any $\boldsymbol{y} \in X$, let

$$
\boldsymbol{y}=\left(z_{1}, \mathrm{e}^{i c x} z_{2}, z_{3}\right), \quad z_{2}=z_{21}+i z_{22}, \quad z_{21}=\operatorname{Re} z_{2}
$$

then

$$
\begin{aligned}
\left\langle H_{\omega, c} \boldsymbol{y}, \boldsymbol{y}\right\rangle & =\operatorname{Re} \int_{R}\left\{\left(-\frac{\partial}{\partial x^{2}}+M^{2}-(p-1)|\varphi|^{p-2}|\psi|^{p}\right) z_{1}^{2}\right. \\
& -\frac{p}{2}|\varphi \psi|^{p-2} \varphi\left(\psi \bar{z}_{2}+\bar{\psi} z_{2}\right) z_{1}-c z_{3 x} z_{1} \\
& -p|\varphi \psi|^{p-2} \varphi \psi z_{1} \bar{z}_{2}+\left[-\frac{1}{2} \frac{\partial}{\partial x^{2}}-\frac{p}{2}|\varphi|^{p}|\psi|^{p-2}\right. \\
& \left.-\frac{p-2}{2}|\varphi|^{p}|\psi|^{p-4} \psi^{2}+i c \frac{\partial}{\partial x}+\omega\right] z_{2} \bar{z}_{2} \\
& \left.+c z_{1 x} z_{3}+z_{3}^{2}\right\} \mathrm{d} x \\
& =\int_{R}\left[\left(1-c^{2}\right) z_{1 x}^{2}+\left(c z_{1 x}+z_{3}\right)^{2}\right. \\
& +\left(M{ }^{2}-(p-1)|\phi|^{p-2}|\psi|^{p}\right) z_{1}^{2} \\
& \left.-2 p|\varphi \psi|^{p-1} z_{1} z_{21}\right] \mathrm{d} x+\left\langle L_{1} z_{21}, z_{21}\right\rangle+\left\langle L_{2} z_{22}, z_{22}\right\rangle \\
& =\int_{R}\left[\left(c z_{1 x}+z_{3}\right)^{2}+p|\varphi \psi|^{p-2}\left(|\psi| z_{1}-|\phi| z_{21}\right)^{2}\right. \\
& \left.+L z_{1}^{2}\right] \mathrm{d} x+\left\langle\bar{L}_{1} z_{21}, z_{21}\right\rangle+\left\langle L_{2} z_{22}, z_{22}\right\rangle \\
&
\end{aligned}
$$

where

$$
\begin{gathered}
L=-\left(1-c^{2}\right) \frac{\partial}{\partial x^{2}}+M^{2}-(2 p-1)|\varphi|^{p-2}|\psi|^{p} \\
L_{1}=-\frac{1}{2} \frac{\partial}{\partial x^{2}}-(p-1)|\varphi|^{p}|\psi|^{p-2}+\omega-\frac{c^{2}}{2} \\
\bar{L}_{1}=-\frac{1}{2} \frac{\partial}{\partial x^{2}}-(p-1)|\varphi|^{p}|\psi|^{p-2}+\omega-\frac{c^{2}}{2}-p|\varphi|^{p}|\psi|^{p-2} \\
L_{2}=-\frac{1}{2} \frac{\partial}{\partial x^{2}}-|\varphi|^{p}|\psi|^{p-2}+\omega-\frac{c^{2}}{2}
\end{gathered}
$$

Since $2 \omega-c^{2}>0$, note that

$$
\begin{aligned}
& \bar{L}_{1}=-\frac{1}{2} \frac{\partial}{\partial x^{2}}+\omega-\frac{c^{2}}{2}+M_{1}(x) \\
& L_{2}=-\frac{1}{2} \frac{\partial}{\partial x^{2}}+\omega-\frac{c^{2}}{2}+M_{2}(x)
\end{aligned}
$$

with

$$
M_{1}(x) \rightarrow 0 \text {, as }|x| \rightarrow \infty ; M_{2}(x) \rightarrow 0, \text { as }|x| \rightarrow \infty
$$

Thus, by Weyl's theorem on the essential spectrum (see [5]), we have

$$
\begin{aligned}
& \sigma_{e s s}\left(\bar{L}_{1}\right)=\left[\omega-\frac{c^{2}}{2},+\infty\right), \omega-\frac{c^{2}}{2}>0 \\
& \sigma_{e s s}\left(L_{2}\right)=\left[\omega-\frac{c^{2}}{2},+\infty\right), \omega-\frac{c^{2}}{2}>0
\end{aligned}
$$

Following from (2.3)-(2.5)

$$
\bar{L}_{1} u^{\prime}=0, L_{2} u=0
$$

By (2.6) and (3.27), we see that $u^{\prime}$ has a simple zero at $x=0$, then Sturm-Liouvill theorem implies that 0 is the second eigenvalue of $\bar{L}_{1}$, and $\bar{L}_{1}$ has exactly one strictly negative eigenvalue $-\lambda^{2}$, with an eigenfunction $\chi_{1}$.

In virtue of (3.24)-(3.27), as in [3], we have the following lemma.

Lemma 2. For any real functions $z_{21} \in H^{1}(R)$, satisfying

$$
\left\langle z_{21}, \chi_{1}\right\rangle=\left\langle z_{21}, u^{\prime}\right\rangle=0
$$

there exists a positive number $\delta_{1}>0$ such that

$$
\left\langle\bar{L}_{1} z_{21}, z_{21}\right\rangle \geq \delta_{1}\left\|z_{21}\right\|_{H^{1}}^{2}
$$

Lemma 3. For any real functions $z_{22} \in H^{1}(R)$, satisfying $\left\langle z_{22}, u\right\rangle=0$, there exists a positive number 
$\delta_{2}>0$ such that

$$
\left\langle L_{2} z_{22}, z_{22}\right\rangle \geq \delta_{2}\left\|z_{22}\right\|_{H^{1}}^{2}
$$

For any $\boldsymbol{y}=\left(z_{1}, \mathrm{e}^{i c x} z_{2}, z_{3}\right), z_{2}=z_{21}+i z_{22}$ We can simply denote by $\boldsymbol{y}=\left(z_{1}, z_{21}, z_{22}, z_{3}\right)$

Choose

$$
\left.\begin{array}{rl}
\boldsymbol{y}_{-}=\left(\frac{p(u v)^{p-1}+\sqrt{p^{2}(u v)^{2 p-2}-\left(p(u v)^{p-2} u^{2}+L\right)}}{p(u v)^{p-2} u^{2}+L} \chi_{1},\right. \\
\chi_{1}, 0,-c z_{1}
\end{array}\right)
$$

then

$$
\left\langle H_{\omega, c} \boldsymbol{y}_{-}, \boldsymbol{y}_{-}\right\rangle=-\lambda^{2}\left\langle\chi_{1}, \chi_{1}\right\rangle<0
$$

Also note that the kernel of $H_{\omega, c}$ is spanned by the following two vectors:

$$
\boldsymbol{y}_{0,1}=\left(-v_{x}, u_{x}, c u,-n_{x}\right), \quad \boldsymbol{y}_{0,2}=(0,0, u, 0)
$$

Let

$$
\begin{aligned}
N= & \left\{k \boldsymbol{y}_{-} / k \in R\right\} \\
Z= & \left\{k_{1} \boldsymbol{y}_{0,1}+k_{2} \boldsymbol{y}_{0,2} / k_{1}, k_{2} \in R\right\} \\
P= & \left\{\boldsymbol{p} \in X / \boldsymbol{p}=\left(p_{1}, p_{2}, p_{3}, p_{4}\right),\right. \\
& \left.\left\langle p_{2}, \chi_{1}\right\rangle=\left\langle p_{2}, u^{\prime}\right\rangle=\left\langle p_{3}, u\right\rangle=0\right\}
\end{aligned}
$$

Lemma 4. For any $\boldsymbol{p} \in P$, defined by (3.32), there exists a constant $\delta>0$ such that

$$
\left\langle H_{\omega, c} \boldsymbol{p}, \boldsymbol{p}\right\rangle \geq \delta\|p\|_{X}^{2}
$$

with $\delta$ independent of $\boldsymbol{p}$.

For any $\boldsymbol{u} \in X, \boldsymbol{u}=\left(z_{1}, z_{21}, z_{22}, z_{3}\right)$

$$
a=\left\langle z_{21}, z_{21}\right\rangle, b_{1}=\frac{\left\langle z_{21}, u^{\prime}\right\rangle}{\left\langle u^{\prime}, u^{\prime}\right\rangle}, b_{2}=\frac{\left\langle z_{22}, u\right\rangle}{\langle u, u\rangle}
$$

then $\boldsymbol{u}=a \boldsymbol{y}_{-}+b_{1} \boldsymbol{y}_{0,1}+b_{2} \boldsymbol{y}_{0,2}+\boldsymbol{p}$.

Thus under the condition of (2.7), Assumption 1 hold and $n\left(H_{\omega, c}\right)=1$.

In the following, we shall verify that $p\left(d^{\prime \prime}\right)=n\left(H_{\omega, c}\right)=1$ under the condition of theorem 1 .

From

$$
d(\omega, c)=E\left(\phi_{\omega, c}\right)-c Q_{1}\left(\phi_{\omega, c}\right)-\omega Q_{2}\left(\phi_{\omega, c}\right)
$$

we have

$$
\begin{aligned}
d_{\omega} & =-Q_{2}\left(\Phi_{\omega, c}\right)=\frac{1}{2} \int_{R} u^{2} \mathrm{~d} x=\frac{c_{1}^{2}}{2 c_{2}} \int_{R} \operatorname{sech}^{\frac{2}{p-1}} x \mathrm{~d} x \\
d_{c} & =-Q_{1}\left(\Phi_{\omega, c}\right)=-\frac{c}{2} \int_{R}\left(u^{2}+2 v^{\prime 2}\right) \mathrm{d} x \\
& =-\frac{c}{2}\left[\frac{c_{1}^{2}}{c_{2}} \int_{R} \operatorname{sech}^{\frac{2}{p-1}} x \mathrm{~d} x+\frac{c_{1}^{2} c_{2}}{\left(1-c^{2}\right)(p-1)^{2}}\right. \\
& \left.\cdot\left(\int_{R} \operatorname{sech}^{\frac{2}{p-1}} x \mathrm{~d} x-\int_{R} \operatorname{sech}^{\frac{2}{p-1}+2} x \mathrm{~d} x\right)\right]
\end{aligned}
$$

Let

$$
\int_{R} \operatorname{sech}^{\frac{2}{p-1}} x \mathrm{~d} x=A>0
$$

then $\int_{R} \operatorname{sech}^{\frac{2}{p-1}+2} x \mathrm{~d} x=\frac{2}{p+1} A>0$.

Thus

$$
\begin{aligned}
d_{\omega c} & =A \frac{\partial}{\partial c}\left(\frac{c_{1}^{2}}{2 c_{2}}\right), \quad d_{\omega \omega}=A \frac{\partial}{\partial \omega}\left(\frac{c_{1}^{2}}{2 c_{2}}\right) \\
d_{c c} & =\left[\frac{\partial}{\partial c}\left(\frac{c_{1}^{2}}{2 c_{2}}\right)\right](-c)\left(1+\frac{(p-1)\left(2 \omega-c^{2}\right)}{\left(1-c^{2}\right)(p+1)}\right) A \\
& +\left(A \frac{c_{1}^{2}}{2 c_{2}}\right)\left(-1-\frac{(p-1)\left(2 \omega-c^{2}\right)}{\left(1-c^{2}\right)(p+1)}\right. \\
& \left.-\frac{2 c^{2}(p-1)(2 \omega-1)}{(p+1)\left(1-c^{2}\right)^{2}}\right)
\end{aligned}
$$

$$
\begin{aligned}
d_{c \omega} & =\left[\frac{\partial}{\partial \omega}\left(\frac{c_{1}^{2}}{2 c_{2}}\right)\right](-c)\left(1+\frac{(p-1)\left(2 \omega-c^{2}\right)}{\left(1-c^{2}\right)(p+1)}\right) A \\
& -\left(A \frac{c_{1}^{2}}{2 c_{2}}\right) \frac{2 c(p-1)}{\left(1-c^{2}\right)(p+1)}
\end{aligned}
$$

Therefore, we obtain

$$
d^{\prime \prime}=\left(\begin{array}{ll}
d_{\omega \omega} & d_{\omega c} \\
d_{c \omega} & d_{c c}
\end{array}\right)
$$

For $1<p<3$, 


$$
\begin{aligned}
& \operatorname{det}\left(d^{\prime \prime}\right)=d_{\omega \omega} d_{c c}-d_{\omega c} d_{c \omega} \\
&= {\left[A \frac{\partial}{\partial \omega}\left(\frac{c_{1}^{2}}{2 c_{2}}\right)\right]\left[\left(A \frac{c_{1}^{2}}{2 c_{2}}\right)\right.} \\
& \cdot\left.\left(-1-\frac{(p-1)\left(2 \omega-c^{2}\right)}{\left(1-c^{2}\right)(p+1)}-\frac{2 c^{2}(p-1)(2 \omega-1)}{(p-1)\left(1-c^{2}\right)^{2}}\right)\right] \\
&+ {\left[A \frac{\partial}{\partial c}\left(\frac{c_{1}^{2}}{2 c_{2}}\right)\right]\left[\left(A \frac{c_{1}^{2}}{2 c_{2}}\right) \frac{2 c(p-1)}{\left(1-c^{2}\right)(p+1)}\right] } \\
& \therefore p\left(d^{\prime \prime}\right)=1 . \\
& \therefore n\left(H_{\omega, c}\right)=p\left(d^{\prime \prime}\right)=1
\end{aligned}
$$

Thus, theorem 2 is proved completely.

\section{References}

[1] M .Grillakis, J. Shatah and W. Strauss, "Stability Theory of Solitary Waves in the Presence of Symmetry, I ," Journal of Functional Analysis, Vol. 74, No. 1, 1987, pp. 160-197. doi:10.1016/0022-1236(87)90044-9

[2] M. Grillakis, J. Shatah and W. Strauss, "Stability Theory of Solitary Waves in the Presence of Symmetry, II," Journal of Functional Analysis, Vol. 94, No. 2, 1990, pp. 308-348.

[3] I. Fukuda and M. Tsumi, "On Coupled Klein-GordonSchrödinger Equations," Journal of Applied Mathematics, Vol. 66, 1978, pp. 358-378.

[4] J. P. Albert and J. L. Bona, "Total Positivity and the Stability of Internal Waves in Stratified Fluids of Finite Depth,” IMA Journal of Applied Mathematics, Vol. 46, No. 1-2, 1991, pp. 1-19. doi:10.1093/imamat/46.1-2.1

[5] M. Reed and B. Simon, "Methods of Modern Mathematical Physics: Fourier Analysis, Self-Adjointness," Academic Press, Waltham, 1975.

[6] B. L. Guo and L. Chen, "Orbital Stability of Solitary Waves of the Long Wave-Short Wave Resonance Equations," Mathematical Methods in the Applied Sciences, Vol. 21, No. 10, 1998, pp. 883-894. 